International Journal of Dentistry and Oral Science (1JDOS)

ISSN: 2377-8075

\title{
Substance P and Neuropeptide Y Expression in Dental Pulp Following Traumatic Injuries
}

\author{
Kathleen G. Neiva ${ }^{1 *}$, Hal I. Coe Jr ${ }^{2}$, Daniella Peinado ${ }^{2}$, Heather Sorenson ${ }^{3}$, Uma P. Nair ${ }^{1}$, Shannon M. Wallet ${ }^{4}$, Roberta Pileggi ${ }^{5}$ \\ ${ }^{1}$ Assistant Professor, Department of Endodontics, University of Florida College of Dentistry, Gainesville, USA. \\ ${ }^{2}$ Graduate Student, Department of Endodontics, University of Florida College of Dentistry, Gainesville, USA. \\ ${ }^{3}$ Biological Scientist/Lab Manager. Department of Oral Biology, University of Florida College of Dentistry, Gainesville, USA. \\ ${ }^{4}$ Associate Professor, Department of Oral Biology, University of Florida College of Dentistry, Gainesville, USA. \\ ${ }^{5}$ Associate Professor, Department of Endodontics, University of Florida College of Dentistry, Gainesville, USA.
}

Research Article

\begin{abstract}
Luxations are the most common and avulsion is the most serious of all traumatic dental injuries. It is well established that neuropeptides play a role in the inflammatory response. However, the presence of neuropeptides in the dental pulp following trauma have not been established.

Objective: To evaluate the expression of Substance P (SP) and Neuropeptide Y (NPY) in the dental pulp following different traumatic injuries (subluxation, lateral luxation, and avulsion).

Materials and Methods: Following endodontic access, samples were collected from the pulp of patients receiving root canal therapy after traumatic injury $(n=43)$. Pulps from healthy teeth extracted for orthodontic reasons were used as control $(\mathrm{n}=10)$. SP and NPY expression was determined by ELISA. Results were analyzed using Student's T test $(\mathrm{p}<0.0001)$ or One-Way ANOVA with Dunn's correction $(\mathrm{p}<0.05)$.

Results: Avulsion demonstrated higher expression of SP and NPY than subluxation and lateral luxation regardless of the pulpal status. Subluxation and lateral luxation demonstrated similar levels of SP and NPY. However, when the pulpal diagnosis was taken in consideration, irreversible pulpitis showed higher levels than necrotic pulps, and lateral luxation showed higher expression of SP and NPY than subluxation.

Conclusions: Our results showed that avulsion presents higher expression of SP and NPY than subluxation and lateral luxation. Pulpal diagnosis correlates with SP and NPY expression in cases of subluxation and lateral luxation, showing higher levels in irreversible pulpitis.
\end{abstract}

Keywords: Neuropeptides; Substance P; Neuropeptide Y; Dental Trauma.

\section{*Corresponding Author:}

Kathleen G. Neiva, DDS, PhD,

Assistant Professor, Department of Endodontics, University of Florida College of Dentistry, Gainesville, FL, 32610, USA.

E-mail:kneiva@dental.ufl.edu

Received: May 23, 2015

Accepted: July 01, 2015

Published: July 02, 2015

Citation: Kathleen G. Neiva et al., (2015) Substance P and Neuropeptide Y Expression in Dental Pulp Following Traumatic Injuries. Int J Dentistry Oral Sci. 2(7), 97-101. doi: http://dx.doi.org/10.19070/2377-80751500021

Copyright: Kathleen G. Neiva ${ }^{\circ}$ 2015. This is an open-access article distributed under the terms of the Creative Commons Attribution License, which permits unrestricted use, distribution and reproduction in any medium, provided the original author and source are credited.

\section{Introduction}

Traumatic injuries can often lead to pulpal inflammation and/ or necrosis. Pulpal inflammation is a complex process involving a wide variety of nervous and vascular reactions, which are key components of the inflammation that could lead to pulp necrosis [1]. Neuropeptides play an active role during neurogenic inflam- mation of the pulp, controlling its blood flow and regulating later stages of inflammation [2].

Neuropeptides are peptide neurotransmitters or neuromodulators synthesized and released from neurons. They trigger biologic effects by activating receptors located on the plasma membrane of target cells. Neuropeptides are widely distributed throughout the body and are present in every branch of the nervous system [3-5]. They have multiple and variable functions, because they can act as growth factors, hormones, and immune modulators. It is generally accepted that the nervous system contributes to the pathophysiology of peripheral inflammation and many inflammatory diseases, including oral diseases such as periodontitis and pulpitis [6].

Substance P (SP) was the first neuropeptide to be identified in dental tissues [7]. Thereafter, immunohistochemical studies described the relative abundance of SP nerve fibers within the dental pulp $[3,4]$. SP is capable of triggering vasodilation, plasma extravasation, immune system activation, chemotaxis, and recruitment and/or regulation of inflammatory cells such as macrophages, mast cells, and lymphocytes [8]. SP has also been implicated in periodontal ligament inflammatory processes [9]. In the dental pulp, it has been reported that SP expression increases with caries progression and with thermal, chemical, and mechanical noxious stimulation [7, 10-14]. 
Immunohistochemical studies have also demonstrated the presence of Neuropeptide Y (NPY) in human dental pulp from sympathetic nerves originating in the superior cervical ganglion [15, 16]. Release of NPY can be induced by stress, physical exercise, myocardial ischemia, and life-threatening situations [17]. Caries and thermal or mechanical irritants of the pulp dentin complex have also been shown to stimulate NPY release [18]. Electrical stimulation of sympathetic nerve fibers causes vasoconstriction and lowers the tissue fluid pressure from the release of NPY in pulp tissues $[17,18]$.

Traumatic injuries produce a mechanical stimulus to the pulp. It is well established that neuropeptides such as SP and NPY play a significant role in pulpal inflammation. It is also well known that after a traumatic injury, the dental pulp can return to its normal condition or develop inflammation and/or necrosis. However the mechanisms and mediators regulating the fate of the dental pulp following trauma is not understood. Since neuropeptides play an important role regulating vasodilation, vasoconstriction, and inflammation, we hypothesized that SP and NPY have different expression levels depending on the severity of the trauma. The expression of SP and NPY in the pulp following traumatic injuries has not been reported. Therefore, the objective of this study was to evaluate the expression of SP and NPY following subluxation, lateral luxation, and avulsion.

\section{Materials and Methods}

\section{Patient selection}

Patients were evaluated at the graduate Endodontic clinic at the University of Florida, College of Dentistry (Gainesville, FL). Patients presented for root canal therapy with history of traumatic tooth injury within two weeks. All patients were considered healthy according to the medical history questionnaire $(n=43)$. A control group consisted of pulpal samples collected from intact teeth extracted for orthodontic reasons $(n=10)$. All patients signed a consent form that was approved, along with the protocol, by the University of Florida Institutional Review Board. Proper type of trauma, pulpal, and periradicular diagnosis were established.

Traumatic injuries included: Subluxation ( $\mathrm{n}=11$, injury to the tooth supporting structures resulting in increased mobility, but without displacement of the tooth), lateral luxation ( $n=21$, displacement of the tooth labial or lingually, characterized by partial or total separation of the periodontal ligament), or avulsion $(n=11$, completely displacement of the tooth out of its socket). Patients included in the avulsion group had history of avulsion and delayed tooth replantation, presenting at a later time for root canal therapy. Pulpal diagnosis was determined using cold and electric pulp tests; and periradicular diagnosis was determined by percussion, palpation, and radiographic evaluation.

Control: Intact maxillary premolars $(n=10)$ were atraumatically extracted for orthodontic reasons and samples were collected from the pulps immediately following extraction, as described below.

Pulpal diagnosis included: Symptomatic irreversible pulpitis (SIP) or necrosis.
Periradicular diagnosis included: Asymptomatic apical periodontitis (AAP) or symptomatic apical periodontitis (SAP).

Exclusion criteria: Patients with medical history of systemic diseases such as diabetes, blood disorder, systemic inflammatory diseases, past radiation treatment, moderate to severe periodontitis, patients taking antibiotics, steroids, or NSAIDs, and patients with non-restorable teeth.

\section{Sample collection}

All samples were collected following the same protocol of crown disinfection, isolation, and endodontic access. Samples were collected when patients presented for root canal treatment, within two weeks following the traumatic injury. Immediately after endodontic access and prior to any irrigation, dental pulp content was collected by placing one Periopaper ${ }^{\mathrm{TM}}$ strip (Oraflow, Smithtown, NY) in the chamber for 15 seconds as previously described [19]. The strips were immediately placed in Eppendorf tubes and stored at $-80^{\circ} \mathrm{C}$ until all the samples were ready to be processed. The samples were then eluted in 200 microliters of PBS and protein concentration was determined using BCA assay. For the control group, samples were collected from the pulp immediately after extraction, following the same protocol.

\section{BCA assay}

Each sample consisted of 200 microliters eluted in PBS. Total protein concentration from each sample was determined using BCA assay according to the manufacturer's instructions (Thermo Scientific Pierce). Briefly, standards were prepared using bovine serum albumin (BSA) and serial dilution; samples were mixed with working reagent, incubated at $37^{\circ} \mathrm{C}$ for 30 minutes, and read in a microplate reader. Protein concentrations were determined in $\mathrm{pg} / \mathrm{ml}$ with reference to standards.

\section{ELISA}

SP and NPY expression was determined using ELISA kits according to manufacturer's instructions ( $\mathrm{R} \& \mathrm{D}$ Systems and Millipore, respectively). Expression levels of SP and NPY was presented in $\mathrm{pg} / \mathrm{ml}$ and normalized by total protein concentration.

\section{Statistical analyses}

Results were analyzed using Student's T test or one-way ANOVA with Dunn's correction $(\mathrm{p}<0.05)$.

\section{Results}

Age of patients varied from 8 to 30 years old, with average of 17 years old. There was no correlation between the age of the patient with the type of trauma, diagnosis, or neuropeptide expression.

SP and NPY expression levels were significantly higher in pulps from teeth with traumatic injury compared with pulps from control teeth $(\mathrm{p}<0.0001$ Student's T test) (Figure $1 \mathrm{a}$ and $1 \mathrm{~b})$.

SP and NPY expression varied according to the type of trauma. Avulsion was the traumatic injury with the highest expression levels of SP and NPY, followed by lateral luxation and subluxation (Figure $2 \mathrm{a}$ and $2 \mathrm{~b}$ ). 
In general, cases of subluxation and lateral luxation had similar expression levels of SP and NPY. However, when the pulpal diagnosis was considered, cases of symptomatic irreversible pulpitis had significantly higher expression of SP and NPY than cases of necrosis. Moreover, samples from lateral luxation had higher expression levels of SP and NPY than those from subluxation (Figure $3 \mathrm{a}$ and $3 \mathrm{~b}$ ).

There was no statistically significant difference in SP and NPY expression when periradicular diagnosis was considered (data not shown).

In summary, the pulpal diagnosis affected SP and NPY expression in subluxation and lateral luxation, being significantly higher in symptomatic irreversible pulpitis than in necrosis (Figure 3). On the other hand, avulsion presented the highest expression levels of SP and NPY even with a necrotic pulpal diagnosis (Figure 2).

\section{Discussion}

The objective of this study was to evaluate the expression of SP and NPY following traumatic injuries in patients compared to controls. Among the neuropeptides expressed in the pulp, we selected SP and NPY because they have been reported to increase with caries progression and with thermal, chemical, and mechanical noxious stimuli $[7,10-14,18]$. In this study, we used Periopaper $^{\mathrm{TM}}$ strips to collect samples from the pulpal chamber of patients the same way it has been demonstrated for gingival crevicular fluid [19]. We analyzed the expression of SP and NPY and correlated it to different types of trauma and pulpal, and periradicular diagnosis.

Our sample size is relatively small since we only included young patients and eliminated traumatic injuries that had occurred more than two weeks from the patient's initial appointment.

Wounding outcomes can differ between different ages; therefore we only included patients up to 30 years old. Moreover, we only added recent cases of trauma since greater lengths of time could result in unreliable variation in neuropeptides expression. We understand that the timing of sample collection following the traumatic injury is a major variable and a limitation of this study. However we included samples collected within two weeks following the trauma because this was the time frame in which the patients presented to our clinic seeking endodontic treatment.

Studies have shown that only about half of the teeth with lateral luxation will become necrotic within the first year, and the other half will remain vital and proceed towards pulp canal obliteration (PCO) [20]. Surprisingly, the majority of the cases included in this study with lateral luxation presented with necrotic pulp. This might be explained by the fact that most of the patients who presented to our clinic were symptomatic and/or had already signs of necrosis. This number of patients might not reflect the entire population who suffered this traumatic injury, but only those who became symptomatic. We speculate that many patients who suf-

Figure 1. (a) SP and (b) NPY expression levels in $\mathrm{pg} / \mathrm{ml}$ in control teeth $(\mathrm{n}=10)$ and teeth with traumatic injury $(\mathrm{n}=43)$. Student's $T$ test ( $\mathrm{p}$ value $<0.0001)$. Asterisks represent significant difference.

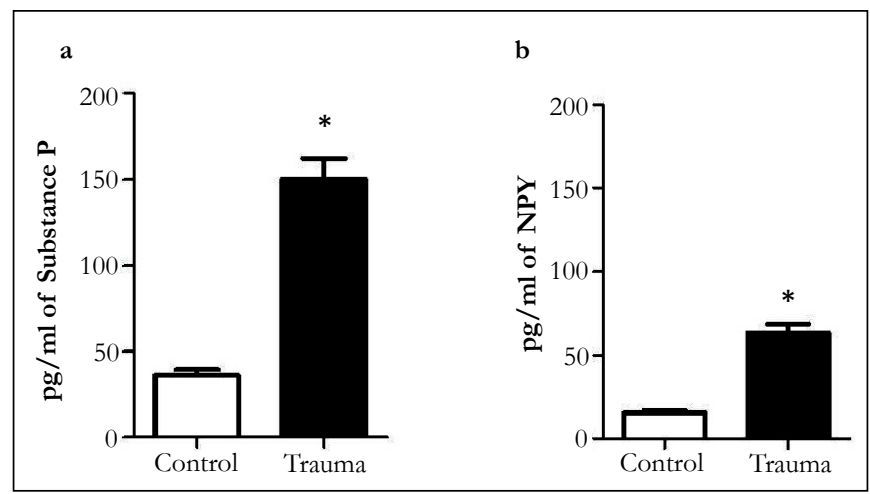

Figure 2. (a) SP and (b) NPY expression levels in $\mathrm{pg} / \mathrm{ml}$ comparing different types of trauma: lateral luxation ( $\mathrm{n}=21)$, subluxation $(\mathrm{n}=11)$, avulsion $(\mathrm{n}=11)$. One Way ANOVA with Dunn's Correction ( $\mathrm{p}$ value $<0.05)$. Bars and asterisks show significant difference between lateral luxation and avulsion, and between subluxation and avulsion.

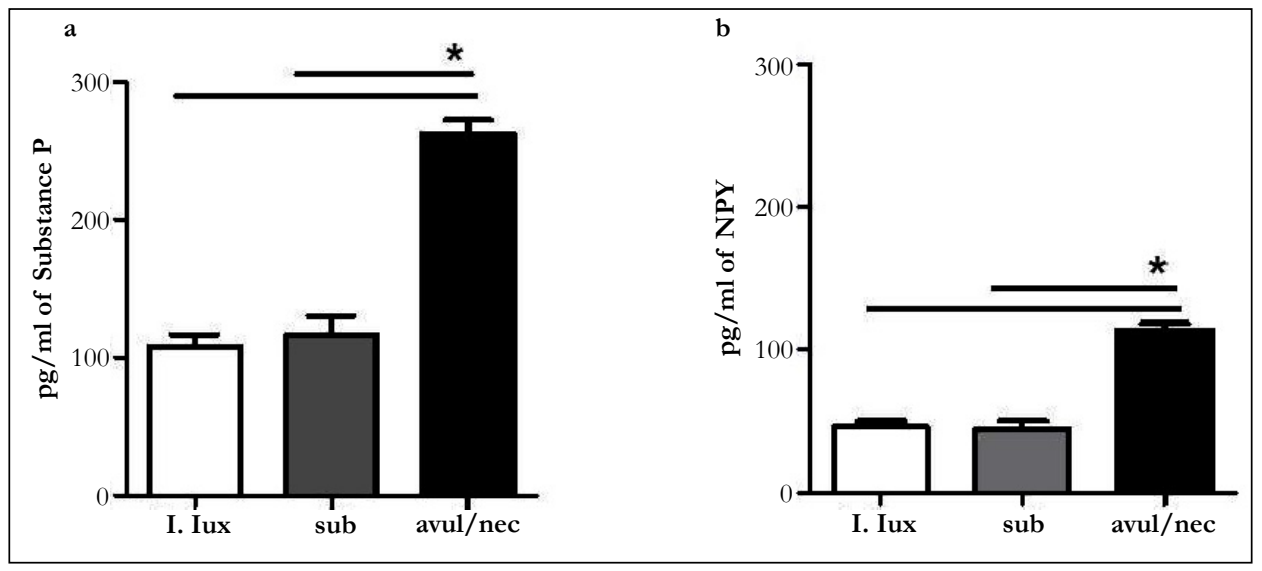


Figure 3. (a) SP and (b) NPY expression levels in $\mathrm{pg} / \mathrm{ml}$ in traumatized teeth according to the pulpal diagnosis: lateral luxation/symptomatic irreversible pulpitis $(n=2)$, lateral luxation/necrosis $(n=19)$, subluxation/symptomatic irreversible pulpitis $(n=8)$, subluxation/necrosis $(n=3)$. One Way ANOVA with Dunn's Correction (p value $<0.05)$. Bars and asterisks show significant difference between 1 . lux/SIP vs. 1 . lux/nec, between 1 . lux/SIP vs. sub/SIP, and between sub/SIP vs. $\mathrm{sub} /$ nec.

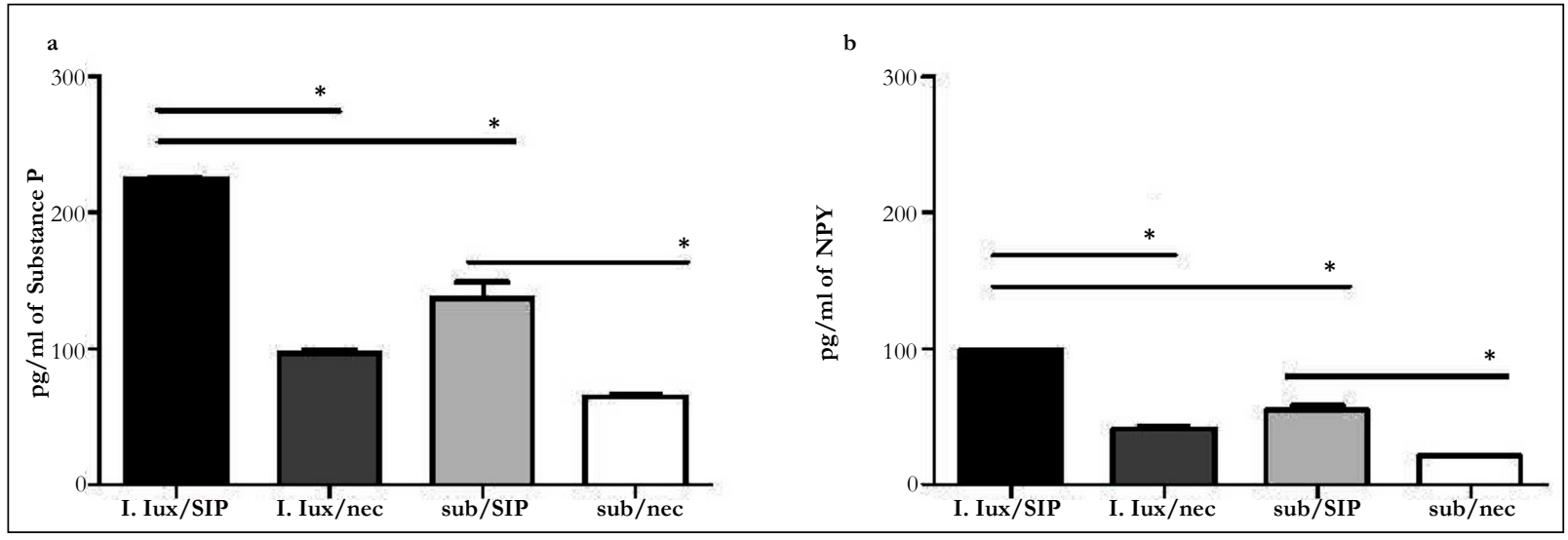

fered lateral luxation have remained asymptomatic and therefore did not seek treatment. It is important to emphasize that teeth with subluxation and lateral luxation were treated because patients were in pain; otherwise teeth were monitored and not included in this study.

Our results demonstrated that SP and NPY expression levels were significantly higher in teeth with traumatic injury compared with normal teeth control, which is in accordance with previous studies that showed a significant higher expression of neuropeptides in inflamed pulps compared with normal pulps [1, 12, 14, 21, 22]. Moreover, SP and NPY expression levels in our control group are comparable with values reported in the literature $[23,24]$.

When we only considered the type of trauma, pulps from teeth with history of avulsion had the highest expression of SP and NPY while lateral luxation and subluxation presented similar levels. However, when we factored in the pulpal diagnosis, pulps from teeth with lateral luxation had higher expression of SP and NPY than subluxation. Moreover, when comparing subluxation and lateral luxation, cases of symptomatic irreversible pulpitis had significantly higher expression of SP and NPY than cases of pulpal necrosis. Notably, avulsion presented significant higher levels of SP and NPY even when the tooth was necrotic. This might be explained by the fact that the pulp necrosis had occurred recently and some remaining tissue was still present in the root canal system; therefore even when teeth tested necrotic, expression of neuropeptides was detectable.

There was no significant difference in SP and NPY expression in teeth with different periradicular diagnosis. SP and NPY levels were similar in asymptomatic and symptomatic apical periodontitis, suggesting that their expression depends mainly on the pulpal diagnosis, and is independent of the periradicular diagnosis.

The higher expression of SP and NPY in teeth with traumatic injury might be explained by the fact that during the inflammatory response, vasodilation of the blood vessels is essential to enable delivery of the appropriate immune system cells to the site of inflammation/infection. Notably, dental innervation can participate directly in the innate immune response in a process termed neurogenic inflammation. In this process, afferent nerves respond to bacterial antigens by release of neuropeptides, which subsequently recruit and activate immune system cells due to the presence of neuropeptide receptors on their cell surfaces $[25,26]$.

This immunoregulation is mediated in part by neuropeptides such as SP and NY. SP can regulate the inflammatory response by altering the function of leukocytes such as chemotaxis, lysosomal enzyme release, and phagocytic activities. The effects of SP on cell-mediated immunity are largely stimulatory, in which synthesis of DNA, protein, and immunoglobulin by mature T and B lymphocytes is significantly enhanced at nanomolar concentrations of this neuropeptide [27]. NPY has been shown to modulate differentiation of T-helper cells, stimulate release of inflammatory mediators from monocytes, and activate natural killer cells [2830]. Sprouting of sensory nerve fibers such as CGRP after dental injury has been demonstrated in the literature [12]. More recently, sprouting of NPY nerve fibers has been reported in the inflamed dental pulp and its significance is not clearly understood [31]. It is known that NPY has an effect on regulation of blood flow and immunomodulation. Since NPY can also act as a vascular growth factor, it may play an important role in revascularization during repair and healing processes in the inflamed dental pulp [32].

The clinical significance of this study is related to the pulpal status following a traumatic injury. Avulsed teeth will most likely develop pulp necrosis, while subluxation and lateral luxation might remain normal, or develop pulpitis or necrosis depending on the severity of the trauma and the pulpal blood flow, which is regulated by SP and NPY expression.

\section{Conclusion}

Considering the limitations of this study, our results showed that pulps from avulsed teeth presented the highest SP and NPY. Subluxation and lateral luxation showed similar levels of SP and NPY; however when we considered the pulpal diagnosis, irreversible pulpitis showed higher levels than necrotic pulps, and lateral luxation showed higher expression of SP and NPY than subluxation. Taken together, these results suggest that the more severe the trauma (avulsion), the higher the expression of SP and NPY. Our data also suggest a correlation between the pulpal diagnosis and SP and NPY expression in cases of less severe traumatic injury (subluxation and lateral luxation). 


\section{Acknowledgement and Declarations}

The authors thank the Department of Endodontics, University of Florida College of Dentistry for the financial support for this project.

The authors deny any conflicts of interest related to this study.

\section{References}

[1]. Kim S (1990) Neurovascular interactions in the dental pulp in health and inflammation. J Endod 16(2): 48-53.

[2]. Olgart L (1996) Neural control of pulpal blood flow. Crit Rev Oral Biol Med 7(2): 159-171.

[3]. Fallgren B, Ekblad E, Edvinsson L (1989) Co-existence of neuropeptides and differential inhibition of vasodilator responses by neuropeptide $\mathrm{Y}$ in guinea pig uterine arteries. Neurosci Lett 100(1-3): 71-76.

[4]. Lee LY, Lou YP, Hong JL, Lundberg JM (1995) Cigarette smoke-induced bronchoconstriction and release of tachykinins in guinea pig lungs. Respir Physiol 99(1): 173-181.

[5]. Edvinsson L, Mulder H, Goadsby PJ, Uddman R (1998) Calcitonin generelated peptide and nitric oxide in the trigeminal ganglion: cerebral vasodilatation from trigeminal nerve stimulation involves mainly calcitonin generelated peptide. J Auton Nerv Syst 70(1-2): 15-22.

[6]. Lundy FT, Linden GJ (2004) Neuropeptides and Neurogenic Mechanisms in Oral and Periodontal Inflammation. Crit Rev Oral Biol Med 15(2): 8298.

[7]. Olgart L, Gazelius B, Brodin E, Nilsson G (1977) Release of substance P-like immunoreactivity from the dental pulp. Acta Physiol Scand 101(4): 510512.

[8]. Caviedes-Bucheli J, Munoz HR, Azuero-Holguin MM, Ulate E (2008) Neuropeptides in dental pulp: the silent protagonists. J Endod 34(7): 773-788.

[9]. Bartold PM, Kylstra A, Lawson R (1994) Substance P: an immunohistochemical and biochemical study in human gingival tissues. A role for neurogenic inflammation? J Periodontol 65(12): 1113-1121.

[10]. Wakisaka S, Ichikawa H, Nishimoto T, Matsuo S, Yamamoto K, et al. (1984) Substance P-like immunoreactivity in the pulp-dentine zone of human molar teeth demonstrated by indirect immunofluorescence. Arch Oral Biol 29(1): 73-75.

[11]. Wakisaka S, Nishikawa S, Ichikawa H, Matsuo S, Takano Y, et al. (1985) The distribution and origin of substance P-like immunoreactivity in the rat molar pulp and periodontal tissues. Arch Oral Biol 30(11-12): 813-818.

[12]. Byers MR, Taylor PE, Khayat BG, Kimberly CL (1990) Effects of injury and inflammation on pulpal and periapical nerves. J Endodontics 16(2): 78-84.

[13]. Takamori K (2000) A histopathological and immunohistochemical study of dental pulp and pulpal nerve fibers in rats after the cavity preparation using Er:YAG laser. J Endodontics 26(2): 95-99.

[14]. Caviedes-Bucheli J, Correa-Ortiz JA, Garcia LV, Lopez-Torres R, Lombana $\mathrm{N}$, et al (2005) The effect of cavity preparation on substance P expression in human dental pulp. J Endod 31(12): 857-859.

[15]. Luthman J, Luthman D, Hokfelt T (1992) Occurrence and distribution of different neurochemical markers in the human dental pulp. Arch Oral Biol
37(3): 193-208.

[16]. Uddman R, Grunditz T, Sundler F (1984) Neuropeptide Y: occurrence and distribution in dental pulps. Acta Odontol Scand 42(6): 361-365.

[17]. Chronwall BM, Zukowska Z (2004) Neuropeptide Y, ubiquitous and elusive. Peptides 25(3): 359-363.

[18]. El Karim IA, Lamey PJ, Linden GJ, Awawdeh LA, Lundy FT (2006) Cariesinduced changes in the expression of pulpal neuropeptide Y. Eur J Oral Sci 114(2): 133-137.

[19]. Karapanou V, Kempuraj D, Theoharides TC (2008) Interleukin-8 is increased in gingival crevicular fluid from patients with acute pulpitis. J Endod 34(2): 148-151.

[20]. Lauridsen E, Hermann NV, Gerds TA, Ahrensburg SS, Kreiborg S, et al. (2012) Combination injuries 3. The risk of pulp necrosis in permanent teeth with extrusion or lateral luxation and concomitant crown fractures without pulp exposure. Dent Traumatol 28(5): 379-385.

[21]. Awawdeh L, Lundy FT, Shaw C, Lamey PJ, Linden GJ, et al. (2002) Quantitative analysis of substance $\mathrm{P}$, neurokinin $\mathrm{A}$ and calcitonin gene-related peptide in pulp tissue from painful and healthy human teeth. Int Endod J 35(1): 30-36.

[22]. Bowles WR, Withrow JC, Lepinski AM, Hargreaves KM (2003) Tissue levels of immunoreactive substance $P$ are increased in patients with irreversible pulpitis. J Endod 29(4): 265-267.

[23]. Caviedes-Bucheli J, Lombana N, Azuero-Holguin MM, Munoz HR (2006) Quantification of neuropeptides (calcitonin gene-related peptide, substance $\mathrm{P}$, neurokinin A, neuropeptide $\mathrm{Y}$ and vasoactive intestinal polypeptide) expressed in healthy and inflamed human dental pulp. Int Endod J 39(5): 394-400.

[24]. Chavarria-Bolanos D, Martinez-Zumaran A, Lombana N, Flores-Reyes H, Pozos-Guillen A (2014) Expression of substance P, calcitonin gene-related peptide, beta-endorphin and methionine-enkephalin in human dental pulp tissue after orthodontic intrusion: a pilot study. Angle orthod 84(3): 521526.

[25]. Fristad I BA, Byers M (2010) Inflammatory nerve responses in the dental pulp. Endodontic Topics 17(1): 12-41.

[26]. Haug SR, Heyeraas KJ (2006) Modulation of dental inflammation by the sympathetic nervous system. J Dent Res 85(6): 488-495.

[27]. McGillis JP, Organist ML, Payan DG (1987) Substance P and immunoregulation. Fed Proc 46(1): 196-199.

[28]. Bedoui S, Kawamura N, Straub RH, Pabst R, Yamamura T, et al. (2003) Relevance of neuropeptide $\mathrm{Y}$ for the neuroimmune crosstalk. J Neuroimmunol 134(1-2): 1-11.

[29]. Bedoui S, Miyake S, Straub RH, von Horsten S, Yamamura T (2004) More sympathy for autoimmunity with neuropeptide Y? Trends immunol 25(10): 508-512.

[30]. Groneberg DA, Folkerts G, Peiser C, Chung KF, Fischer A (2004) Neuropeptide Y (NPY). Pulmonary pharmacology \& therapeutics 17(4): 173-180.

[31]. Haug SR, Heyeraas KJ (2003) Effects of sympathectomy on experimentally induced pulpal inflammation and periapical lesions in rats. Neuroscience 120(3): 827-836.

[32]. Zukowska-Grojec Z, Karwatowska-Prokopczuk E, Fisher TA, Ji H (1998) Mechanisms of vascular growth-promoting effects of neuropeptide Y: role of its inducible receptors. Regul pept 75-76: 231-238. 